\title{
Correlated Triplet Pair Formation Activated by Geometry Relaxation in Directly Linked Tetracene Dimer (5,5'-Bitetracene)
}

\author{
Katsuyuki Shizu, Chihaya Adachi, and Hironori Kaji*
}

Cite This: ACS Omega 2021, 6, 2638-2643

Read Online

ABSTRACT: Singlet fission (SF) materials have the potential to overcome the traditional external quantum efficiency limits of organic light-emitting diodes (OLEDs). In this study, we theoretically designed an intramolecular SF molecule, 5,5'bitetracene (55BT), in which two tetracene units were directly connected through a $\mathrm{C}-\mathrm{C}$ bond. Using quantum chemical calculation and the Fermi golden rule, we show that 55BT undergoes efficient SF induced by geometry relaxation in a locally excited singlet state, ${ }^{1}\left(\mathrm{~S}_{0} \mathrm{~S}_{1}\right)$. Compared with another highperforming SF system, the tetracene dimer in the crystalline state, 55BT has advantages when used in doped systems owing to covalent bonding of the two tetracene units. This feature makes 55BT a promising candidate triplet sensitizer for near-infrared OLEDs.

\section{INTRODUCTION}

Singlet fission (SF) is a nonradiative decay process that generates two triplet excitons from one singlet exciton. ${ }^{1,2}$ Organic molecules that undergo SF potentially increase the number of triplet excitons and are of great interest for applications as triplet sensitizers for organic optoelectronic devices. $^{3-5}$ Over the past decade, the triplet harvesting effect of SF molecules has been applied to light-conversion devices, such as photodetectors ${ }^{3}$ and organic solar cells. ${ }^{4}$ Recently, the effect has been used to improve the exciton-generation efficiency of near-infrared organic light-emitting diodes (NIR-OLEDs). ${ }^{5}$ Although NIR-OLEDs have attracted great interest because of their potential applications to bioimaging, telecommunication, and night-vision devices, ${ }^{6-8}$ the external quantum efficiencies (EQEs) of NIR-OLEDs remain low compared with those of visible OLEDs. ${ }^{6}$ Most NIR-OLEDs contain phosphorescent molecules as emitters, and hence, their EQE depends on the triplet harvesting efficiency of the host materials. The use of an efficient SF molecule as a triplet host can break the traditional limit of the EQE, increasing the maximum potential to $125 \% .{ }^{5}$ Furthermore, by incorporating a thermally activated delayed fluorescence molecule, the maximum potential EQE may be improved up to $200 \%$. However, studies of SF-based NIR-OLEDs have been limited partially because there are few available SF materials suitable for triplet sensitizers in NIR-OLEDs.

Among these few SF materials, tetracene $(\mathrm{Tc})$ is one of the most commonly studied SF molecules ${ }^{1,2,9-15}$ and its derivatives undergo SF. Examples of Tc-based SF molecules include 5,12-diphenyltetracene, ${ }^{16,17}$ rubrene, ${ }^{18-25}$ and linked

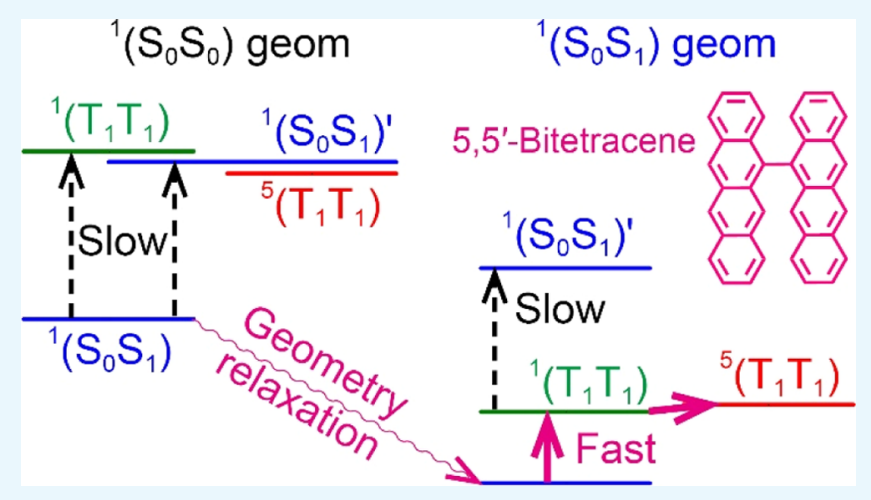

Tc molecules. ${ }^{26-38}$ Tc, 5,12-diphenyltetracene, and rubrene have intermolecular SF, whereas linked Tc molecules have inter- and intramolecular SF. Although Tc derivatives have high SF efficiency, the performance depends on the relative geometry of the two interacting Tc's. From the viewpoint of device fabrication, molecules that have intramolecular SF are advantageous because SF materials are often used in an amorphous state and/or mixed with other materials in devices.

In a previous study, based on quantum chemical calculations and the Fermi golden rule, we theoretically investigated structure-property relationships in the intramolecular SF rates of several Tc-based molecules. ${ }^{39}$ We found that the SF rate of para-phenylene-linked bis(ethynyl tetracene) largely depends on the torsion angle between the two Tc units. In this study, we theoretically designed a 5,5'-bitetracene (55BT, Figure 1), in which two Tc units are directly linked by a $\mathrm{C}-\mathrm{C}$ bond. The torsion angle between the Tc units is expected to be a large well-defined value because of steric repulsion between hydrogen atoms bonded to $4 / 6$ and $4^{\prime} / 6^{\prime}$ carbon atoms (Figure 1). Hence, 55BT is a prototypical molecule for studies of intramolecular SF. However, despite the simple molecular structure without any covalent linker and substituent, intra-

Received: October 1, 2020

Accepted: November 17, 2020

Published: December 1, 2020 


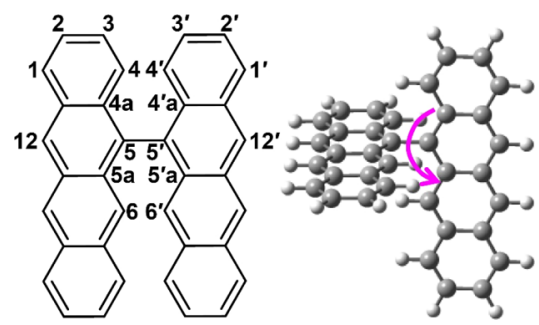

Figure 1. Chemical structure of 55BT with an atom labeling scheme for carbon atoms and ball-and-stick representation of the optimized ${ }^{1}\left(\mathrm{~S}_{0} \mathrm{~S}_{1}\right)$ geometry of 55BT calculated at the TD- $\omega$ B97X-D/6-31G(d) level of theory. The pink arrow shows the torsion angle between the two Tc units.

molecular SF in 55BT has not been investigated. Furthermore, as far as we are aware, there have been no reports describing its synthetic routes and photophysical, electrochemical, and thermodynamic properties. Toyota et al. reported that they failed to synthesize 55BT but successfully obtained 55BT derivatives by introducing triisopropylsilylethynyl groups into the 12 and $12^{\prime}$ carbon atoms. $^{40}$

\section{RESULTS AND DISCUSSION}

Figure $\mathrm{S} 1$ compares optimized ${ }^{1}\left(\mathrm{~S}_{0} \mathrm{~S}_{0}\right),{ }^{1}\left(\mathrm{~S}_{0} \mathrm{~S}_{1}\right)$, and ${ }^{5}\left(\mathrm{~T}_{1} \mathrm{~T}_{1}\right)$ geometries of 55BT with optimized $S_{0}, S_{1}$, and $T_{1}$ geometries of the isolated Tc. The method of geometry optimization is described in detail in the Computational Methods section. From the calculated $\mathrm{C}-\mathrm{C}$ bond lengths (Figure $\mathrm{S} 1$ ), in the ${ }^{1}\left(S_{0} S_{0}\right)-{ }^{5}\left(T_{1} T_{1}\right)$ geometry, the individual $T c$ units have character of the $S_{0} / T_{1}$ geometry of the isolated Tc. In the ${ }^{1}\left(S_{0} S_{1}\right)$ geometry, one of the Tc units has the character of the $\mathrm{S}_{0}$ geometry of the isolated $\mathrm{Tc}$, whereas the other has the character of the $S_{1}$ geometry. The torsion angles were calculated to be $89.7,82.7$, and $88.4^{\circ}$ for the ${ }^{1}\left(S_{0} S_{0}\right)$, ${ }^{1}\left(\mathrm{~S}_{0} \mathrm{~S}_{1}\right)$, and ${ }^{5}\left(\mathrm{~T}_{1} \mathrm{~T}_{1}\right)$ geometries, respectively (Figure $\mathrm{S} 1$ ), and their difference was at most $7^{\circ}$. Figure $S 2$ shows a complete energy diagram containing the ${ }^{1}\left(\mathrm{~S}_{0} \mathrm{~S}_{0}\right),{ }^{1}\left(\mathrm{~S}_{0} \mathrm{~S}_{1}\right)$, and ${ }^{5}\left(\mathrm{~T}_{1} \mathrm{~T}_{1}\right)$ geometries. From Figure $S 2$, the energy difference between ${ }^{1}\left(\mathrm{~S}_{0} \mathrm{~S}_{1}\right)$ and ${ }^{1}\left(\mathrm{~T}_{1} \mathrm{~T}_{1}\right)$ largely depends on the molecular geometry, whereas the energy difference between ${ }^{1}\left(\mathrm{~T}_{1} \mathrm{~T}_{1}\right)$ and ${ }^{5}\left(\mathrm{~T}_{1} \mathrm{~T}_{1}\right)$ is smaller than $k_{\mathrm{B}} \mathrm{T}$ at room temperature $(26 \mathrm{meV})$, irrespective of the molecular geometry (Table S12). Thus, molecular geometry affects SF efficiency by varying the ${ }^{1}\left(\mathrm{~S}_{0} \mathrm{~S}_{1}\right)-{ }^{1}\left(\mathrm{~T}_{1} \mathrm{~T}_{1}\right)$ energy difference.

Figure 2 shows a state-energy diagram and transition rates calculated at the ${ }^{1}\left(\mathrm{~S}_{0} \mathrm{~S}_{0}\right)$ and ${ }^{1}\left(\mathrm{~S}_{0} \mathrm{~S}_{1}\right)$ geometries. We assume that $\mathrm{SF}$ is initiated by photoexcitation of ${ }^{1}\left(\mathrm{~S}_{0} \mathrm{~S}_{0}\right)$ at the ${ }^{1}\left(\mathrm{~S}_{0} \mathrm{~S}_{0}\right)$ geometry. Because the transition dipole moment between ${ }^{1}\left(\mathrm{~S}_{0} \mathrm{~S}_{0}\right)$ and ${ }^{1}\left(\mathrm{~S}_{0} \mathrm{~S}_{1}\right)$ is large (3.0478 debye, Table $\left.\mathrm{S} 7\right)$, it is reasonable to consider that ${ }^{1}\left(\mathrm{~S}_{0} \mathrm{~S}_{1}\right)$ is the initial excited state of SF. After formation of ${ }^{1}\left(S_{0} S_{1}\right)$ by photoexcitation, ${ }^{1}\left(S_{0} S_{1}\right)$ will be nonradiatively converted into ${ }^{1}\left(S_{0} S_{0}\right),{ }^{1}\left(S_{0} S_{1}\right)^{\prime}$, or ${ }^{1}\left(T_{1} T_{1}\right)$ via internal conversion (IC). Alternatively, for a radiative process, ${ }^{1}\left(S_{0} S_{1}\right)$ will be converted into ${ }^{1}\left(S_{0} S_{0}\right)$, resulting in fluorescence. Calculated ${ }^{1}\left(\mathrm{~S}_{0} \mathrm{~S}_{1}\right) \rightarrow{ }^{1}\left(\mathrm{~S}_{0} \mathrm{~S}_{0}\right),{ }^{1}\left(\mathrm{~S}_{0} \mathrm{~S}_{1}\right) \rightarrow$ ${ }^{1}\left(S_{0} S_{1}\right)^{\prime}$, and ${ }^{1}\left(S_{0} S_{1}\right) \rightarrow{ }^{1}\left(T_{1} T_{1}\right)$ nonradiative transition rates and fluorescence rate were $1.8 \times 10^{4}, 1.1 \times 10^{10}, 2.7 \times 10^{7}$, and $1.4 \times 10^{9} \mathrm{~s}^{-1}$, respectively, and therefore, the ${ }^{1}\left(\mathrm{~S}_{0} \mathrm{~S}_{1}\right) \rightarrow$ ${ }^{1}\left(S_{0} S_{1}\right)^{\prime}$ excitation is the most probable interstate transition among them. Another possible decay channel from ${ }^{1}\left(S_{0} S_{1}\right)$ is the geometry relaxation (vibrational relaxation) that deforms

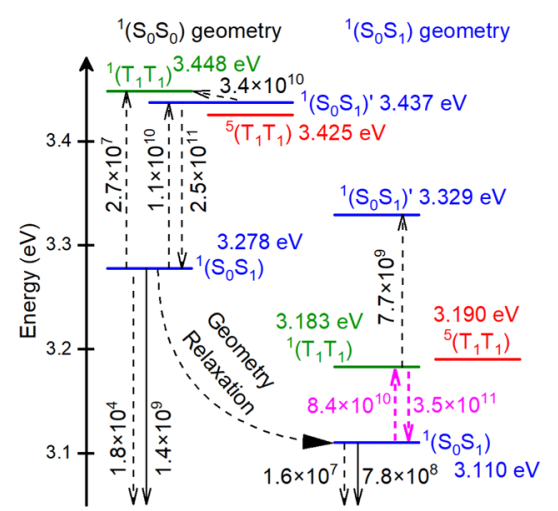

Figure 2. Energy-level diagram and transition rates $\left(\mathrm{s}^{-1}\right)$ calculated at the ${ }^{1}\left(\mathrm{~S}_{0} \mathrm{~S}_{0}\right)$ and ${ }^{1}\left(\mathrm{~S}_{0} \mathrm{~S}_{1}\right)$ geometries. Dashed arrows show nonradiative transitions; solid arrows show radiative transitions; curved arrow shows geometry relaxation in ${ }^{1}\left(S_{0} S_{1}\right)$.

the molecular geometry into the ${ }^{1}\left(\mathrm{~S}_{0} \mathrm{~S}_{1}\right)$ geometry. Because the typical frequency of molecular vibrations is $10^{12}$ to $10^{14} \mathrm{~s}^{-141}$ and faster than the ${ }^{1}\left(S_{0} S_{1}\right) \rightarrow{ }^{1}\left(S_{0} S_{1}\right)^{\prime}$ transition rate, the geometry relaxation is the dominant decay channel from ${ }^{1}\left(S_{0} S_{1}\right)$. Some ${ }^{1}\left(S_{0} S_{1}\right)$ can be excited to ${ }^{1}\left(S_{0} S_{1}\right)^{\prime}$ and further excited to ${ }^{1}\left(\mathrm{~T}_{1} \mathrm{~T}_{1}\right)$. However, because calculated ${ }^{1}\left(\mathrm{~S}_{0} \mathrm{~S}_{1}\right)^{\prime} \rightarrow$ ${ }^{1}\left(\mathrm{~S}_{0} \mathrm{~S}_{1}\right)$ and ${ }^{1}\left(\mathrm{~S}_{0} \mathrm{~S}_{1}\right)^{\prime} \rightarrow{ }^{1}\left(\mathrm{~T}_{1} \mathrm{~T}_{1}\right)$ rates are $2.5 \times 10^{11}$ and $3.4 \times$ $10^{10} \mathrm{~s}^{-1}$, respectively, even though ${ }^{1}\left(\mathrm{~S}_{0} \mathrm{~S}_{1}\right)^{\prime}$ is generated, only approximately $10 \%$ of ${ }^{1}\left(S_{0} S_{1}\right)^{\prime}$ is converted into ${ }^{1}\left(T_{1} T_{1}\right)$. Hence, at the ${ }^{1}\left(S_{0} S_{0}\right)$ geometry, geometry relaxation mainly occurs and 55BT does not undergo efficient SF via the ${ }^{1}\left(\mathrm{~S}_{0} \mathrm{~S}_{1}\right)$ $\rightarrow{ }^{1}\left(\mathrm{~T}_{1} \mathrm{~T}_{1}\right)$ nor ${ }^{1}\left(\mathrm{~S}_{0} \mathrm{~S}_{1}\right) \rightarrow{ }^{1}\left(\mathrm{~S}_{0} \mathrm{~S}_{1}\right)^{\prime} \rightarrow{ }^{1}\left(\mathrm{~T}_{1} \mathrm{~T}_{1}\right)$ transitions.

After 55BT deforms to the ${ }^{1}\left(\mathrm{~S}_{0} \mathrm{~S}_{1}\right)$ geometry, ${ }^{1}\left(\mathrm{~S}_{0} \mathrm{~S}_{1}\right)$ can be nonradiatively converted into ${ }^{1}\left(\mathrm{~S}_{0} \mathrm{~S}_{0}\right)$ or ${ }^{1}\left(\mathrm{~T}_{1} \mathrm{~T}_{1}\right)$ or radiatively converted into ${ }^{1}\left(\mathrm{~S}_{0} \mathrm{~S}_{0}\right)$. Calculated ${ }^{1}\left(\mathrm{~S}_{0} \mathrm{~S}_{1}\right) \rightarrow{ }^{1}\left(\mathrm{~S}_{0} \mathrm{~S}_{0}\right)$ and ${ }^{1}\left(\mathrm{~S}_{0} \mathrm{~S}_{1}\right) \rightarrow{ }^{1}\left(\mathrm{~T}_{1} \mathrm{~T}_{1}\right)$ nonradiative transition rates and ${ }^{1}\left(\mathrm{~S}_{0} \mathrm{~S}_{1}\right) \rightarrow$ ${ }^{1}\left(\mathrm{~S}_{0} \mathrm{~S}_{0}\right)$ radiative transition rate were $1.6 \times 10^{7}, 8.4 \times 10^{10}$, and $7.8 \times 10^{8} \mathrm{~s}^{-1}$, respectively (Figure 2$)$. The ${ }^{1}\left(\mathrm{~S}_{0} \mathrm{~S}_{1}\right) \rightarrow{ }^{1}\left(\mathrm{~T}_{1} \mathrm{~T}_{1}\right)$ nonradiative transition rate is several orders of magnitude faster than the other rates and is the dominant transition pathway from ${ }^{1}\left(\mathrm{~S}_{0} \mathrm{~S}_{1}\right)$. The calculated ${ }^{1}\left(\mathrm{~S}_{0} \mathrm{~S}_{1}\right) \rightarrow{ }^{1}\left(\mathrm{~T}_{1} \mathrm{~T}_{1}\right)$ rate of $8.4 \times 10^{10} \mathrm{~s}^{-1}$ is comparable to that calculated for crystalline $\mathrm{Tc}^{42}\left(1.7 \times 10^{11} \mathrm{~s}^{-1}\right)$ but one-order of magnitude smaller than that calculated for a para-phenylene-linked Tc dimer ${ }^{39}(2.1 \times$ $\left.10^{12} \mathrm{~s}^{-1}\right)$. The energy difference between ${ }^{1}\left(\mathrm{~T}_{1} \mathrm{~T}_{1}\right)$ and ${ }^{5}\left(\mathrm{~T}_{1} \mathrm{~T}_{1}\right)$ was calculated to be $7 \mathrm{meV}$, which is smaller than $k_{\mathrm{B}} T$ at the room temperature $(26 \mathrm{meV})$. Hence, if the ${ }^{1}\left(\mathrm{~T}_{1} \mathrm{~T}_{1}\right) \rightarrow$ ${ }^{5}\left(\mathrm{~T}_{1} \mathrm{~T}_{1}\right)$ conversion rate is faster than the ${ }^{1}\left(\mathrm{~S}_{0} \mathrm{~S}_{1}\right) \rightarrow{ }^{1}\left(\mathrm{~S}_{0} \mathrm{~S}_{0}\right)$ radiative transition rate $\left(7.8 \times 10^{8} \mathrm{~s}^{-1}\right),{ }^{5}\left(\mathrm{~T}_{1} \mathrm{~T}_{1}\right)$ is produced efficiently. The ${ }^{5}\left(\mathrm{~T}_{1} \mathrm{~T}_{1}\right)$ formation is followed by geometry relaxation that changes the molecular geometry into the ${ }^{5}\left(\mathrm{~T}_{1} \mathrm{~T}_{1}\right)$ geometry, at which the ${ }^{1}\left(\mathrm{~T}_{1} \mathrm{~T}_{1}\right)$ lies $641 \mathrm{meV}$ lower in energy than ${ }^{1}\left(\mathrm{~S}_{0} \mathrm{~S}_{1}\right)$ (Figure $\left.\mathrm{S} 2\right)$. The large ${ }^{1}\left(\mathrm{~T}_{1} \mathrm{~T}_{1}\right)-{ }^{1}\left(\mathrm{~S}_{0} \mathrm{~S}_{1}\right)$ energy difference results in a small ${ }^{1}\left(\mathrm{~T}_{1} \mathrm{~T}_{1}\right) \rightarrow{ }^{1}\left(\mathrm{~S}_{0} \mathrm{~S}_{1}\right)$ rate of $1.6 \times 10^{3} \mathrm{~s}^{-1}$ at the ${ }^{5}\left(\mathrm{~T}_{1} \mathrm{~T}_{1}\right)$ geometry, and therefore, even though ${ }^{1}\left(\mathrm{~T}_{1} \mathrm{~T}_{1}\right)$ is generated from ${ }^{5}\left(\mathrm{~T}_{1} \mathrm{~T}_{1}\right),{ }^{1}\left(\mathrm{~T}_{1} \mathrm{~T}_{1}\right)$ does not convert back into ${ }^{1}\left(\mathrm{~S}_{0} \mathrm{~S}_{1}\right)$.

The above SF scenario of 55BT is summarized as follows. (i) After photo-excitation of ${ }^{1}\left(S_{0} S_{0}\right)$, the molecular geometry relaxes to the ${ }^{1}\left(S_{0} S_{1}\right)$ geometry. (ii) The ${ }^{1}\left(S_{0} S_{1}\right) \rightarrow{ }^{1}\left(T_{1} T_{1}\right)$ transition occurs efficiently, followed by ${ }^{1}\left(\mathrm{~T}_{1} \mathrm{~T}_{1}\right) \rightarrow{ }^{5}\left(\mathrm{~T}_{1} \mathrm{~T}_{1}\right)$ owing to the very small energy gap of $7 \mathrm{meV}$ between the two (the energy gap is always small irrespective of the geometry, see Figure S2). (iii) The molecular geometry relaxes to the 
${ }^{5}\left(\mathrm{~T}_{1} \mathrm{~T}_{1}\right)$ geometry and finally ${ }^{5}\left(\mathrm{~T}_{1} \mathrm{~T}_{1}\right)$ splits into two $\mathrm{T}_{1}$ states. Here, we discuss the reasons for the fast ${ }^{1}\left(S_{0} S_{1}\right)-{ }^{1}\left(T_{1} T_{1}\right)$ IC rate $\left(3.5 \times 10^{11} \mathrm{~s}^{-1}\right)$ at the ${ }^{1}\left(\mathrm{~S}_{0} \mathrm{~S}_{1}\right)$ geometry from the viewpoint of vibronic coupling. Figure 3 shows calculated $V_{m}$,

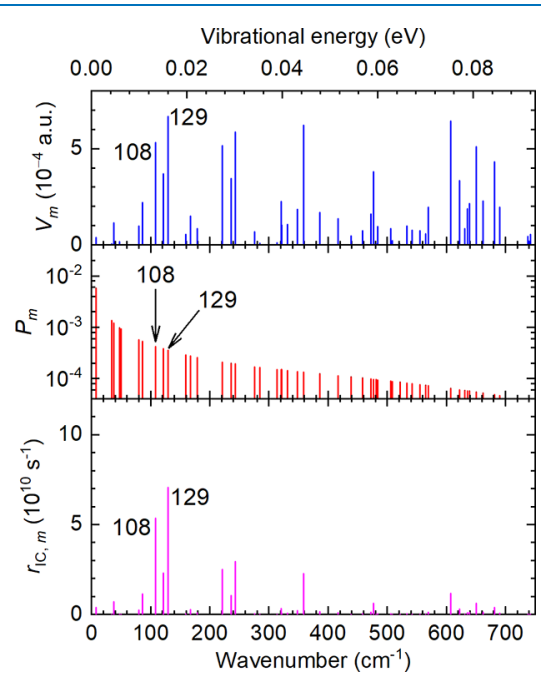

Figure 3. $V_{m}, P_{m}$ and $r_{\mathrm{IC}, m}$ vs wavenumber plots for the ${ }^{1}\left(\mathrm{~S}_{0} \mathrm{~S}_{1}\right) \rightarrow$ ${ }^{1}\left(\mathrm{~T}_{1} \mathrm{~T}_{1}\right)$ transition calculated at the stable ${ }^{1}\left(\mathrm{~S}_{0} \mathrm{~S}_{1}\right)$ geometry. Wavenumbers of the vibrational modes designated by arrows are 108 and $129 \mathrm{~cm}^{-1}$ : four-ring out-of-plane bending $\left(108 \mathrm{~cm}^{-1}\right)$; ringring in-plane bending $\left(129 \mathrm{~cm}^{-1}\right)$.

$P_{m}$, and $r_{\mathrm{IC}, m}$ versus wavenumber plots for the transition (the description of vibrational modes follows that used by Mackie et al. $\left.^{43}\right)$. SF is promoted by vibrational modes, for which the $r_{\mathrm{IC}, m}$ values are large. From eq $1, r_{\mathrm{IC}, m}$ is large when the product of $V_{m}{ }^{2} P_{m}$ is large. Because $P_{m}$ is large for wavenumbers $0-400$ $\mathrm{cm}^{-1}$, the vibrational modes for wavenumbers $<400 \mathrm{~cm}^{-1}$ mainly activate the ${ }^{1}\left(\mathrm{~S}_{0} \mathrm{~S}_{1}\right)-{ }^{1}\left(\mathrm{~T}_{1} \mathrm{~T}_{1}\right)$ IC (Figure 3 ). The sum of $r_{\mathrm{IC}, m}$ of the vibrational modes for wavenumbers $<400 \mathrm{~cm}^{-1}$ was calculated to be $2.7 \times 10^{11} \mathrm{~s}^{-1}$, which accounts for $79 \%$ of the total IC rate of $3.5 \times 10^{11} \mathrm{~s}^{-1}$. The largest and second largest $r_{\mathrm{IC}, m}$ correspond to the low-frequency modes (129 and $\left.108 \mathrm{~cm}^{-1}\right)$. The trend that low-frequency modes activate ${ }^{1}\left(S_{0} S_{1}\right)-{ }^{1}\left(T_{1} T_{1}\right)$ IC is same as that observed for crystalline $T c$.

Next, we discuss the reason for the large $V_{m}$ values of 55BT. As previously reported, ${ }^{39,42} V_{m}$ values are large when the spatial overlap $(\rho)$ between the wave functions of ${ }^{1}\left(\mathrm{~S}_{0} \mathrm{~S}_{1}\right)$ and ${ }^{1}\left(\mathrm{~T}_{1} \mathrm{~T}_{1}\right)$ is large. Figure $\mathrm{S} 4$ shows the highest occupied molecular orbital (HOMO), lowest unoccupied molecular orbital (LUMO), HOMO - 1, and LUMO +1 of the Hartree-Fock determinant calculated at the stable ${ }^{1}\left(\mathrm{~S}_{0} \mathrm{~S}_{1}\right)$ geometry based on the RAS-2SF(4,4)/6-31G(d) method. Reflecting the broken symmetry of the ${ }^{1}\left(\mathrm{~S}_{0} \mathrm{~S}_{1}\right)$ geometry, the HOMO, LUMO, HOMO -1 , and LUMO +1 are localized on one of the Tc units. One would expect that the $\rho$ distribution would be localized because of the localized HOMO, LUMO, HOMO - 1, and LUMO + 1. However, unexpectedly, the $\rho$ distribution is largely delocalized over the two Tc units (Figure S5). This is because ${ }^{1}\left(\mathrm{~T}_{1} \mathrm{~T}_{1}\right)$ is mainly expressed in terms of double electron excitations between the Tc units (e.g., $\Phi_{\mathrm{HOMO}-1 \text { HOMO }}^{\mathrm{LUMO} \text { LUMO }}$ see Table S8): the HOMO - 1 $\rightarrow$ LUMO excitation is a single electron excitation from the left to the right $\mathrm{Tc}$, whereas the $\mathrm{HOMO} \rightarrow \mathrm{LUMO}+1$ excitation is single electron excitation from the right to the left Tc. In this sense, ${ }^{1}\left(T_{1} T_{1}\right)$ can be viewed as having a charge transfer character. Because $\rho$ is largely distributed, various vibrational modes have strong vibronic coupling (Figure 3 ).

The triplet energy of 55BT is important when 55BT is used as a triplet-exciton sensitizer for phosphorescent emitters. If the triplet energy of 55BT is too low, phosphorescent emitters that can accept the triplet energy are limited and 55BT would be useless as a triplet-exciton sensitizer even if 55BT showed efficient SF. Since 55BT has two Tc units, there is a possibility that the triplet energy of 55BT is largely lowered compared with $\mathrm{Tc}$ because of the $\pi$-conjugation over the two Tc units. To exclude the possibility, we calculated the triplet energies of 55BT and Tc (Supporting Information). 55BT was found to have a triplet energy $(1.26 \mathrm{eV}, 983 \mathrm{~nm})$ comparable to that of Tc $(1.29 \mathrm{eV}, 963 \mathrm{~nm})$, suggesting that the $\pi$-conjugation has little influence on the triplet energy.

\section{CONCLUSIONS}

From the restricted-active-space double spin-flip (RAS-2SF) calculations and the Fermi golden rule, we theoretically designed a promising intramolecular SF candidate, 55BT. We predict that 55BT undergoes efficient ${ }^{1}\left(\mathrm{~T}_{1} \mathrm{~T}_{1}\right)$ formation after geometry relaxation to the stable ${ }^{1}\left(\mathrm{~S}_{0} \mathrm{~S}_{1}\right)$ geometry. The largely twisted structure of the 55BT promotes a very fast ${ }^{1}\left(\mathrm{~S}_{0} \mathrm{~S}_{1}\right) \rightarrow$ ${ }^{1}\left(\mathrm{~T}_{1} \mathrm{~T}_{1}\right)$ transition rate based on both the small ${ }^{1}\left(\mathrm{~S}_{0} \mathrm{~S}_{1}\right)-^{1}\left(\mathrm{~T}_{1} \mathrm{~T}_{1}\right)$ energy difference and the large vibronic couplings at the ${ }^{1}\left(\mathrm{~S}_{0} \mathrm{~S}_{1}\right)$ geometry. The calculated ${ }^{1}\left(\mathrm{~S}_{0} \mathrm{~S}_{1}\right) \rightarrow$ ${ }^{1}\left(\mathrm{~T}_{1} \mathrm{~T}_{1}\right)$ transition rate of 55BT is comparable to that of the Tc dimer in the crystalline state, suggesting that the directly connected structure of 55BT is advantageous for realizing efficient intramolecular SF. 55BT is a promising tripletsensitizing SF molecule for developing SF-based NIR-OLEDs.

\section{COMPUTATIONAL METHODS}

To investigate the SF mechanism of 55BT using the Fermi golden rule, we consider the following electronic states: (1) the singlet ground state, ${ }^{1}\left(\mathrm{~S}_{0} \mathrm{~S}_{0}\right)$; (2) two singlet excited states mainly expressed in terms of singly excited electronic states, ${ }^{1}\left(S_{0} S_{1}\right)$ and ${ }^{1}\left(S_{0} S_{1}\right)^{\prime}$, where ${ }^{1}\left(S_{0} S_{1}\right)^{\prime}$ lies higher in energy than ${ }^{1}\left(S_{0} S_{1}\right)$; (3) the singlet excited state mainly expressed in terms of doubly excited electronic states and denoted the correlated triplet pair, ${ }^{1}\left(\mathrm{~T}_{1} \mathrm{~T}_{1}\right)$; and (4) the quintet state, ${ }^{5}\left(\mathrm{~T}_{1} \mathrm{~T}_{1}\right)$. First, we used density functional theory (DFT) and time-dependent DFT for geometry optimization and frequency analysis of ${ }^{1}\left(\mathrm{~S}_{0} \mathrm{~S}_{0}\right),{ }^{1}\left(\mathrm{~S}_{0} \mathrm{~S}_{1}\right)$, and ${ }^{5}\left(\mathrm{~T}_{1} \mathrm{~T}_{1}\right):$ for ${ }^{1}\left(\mathrm{~S}_{0} \mathrm{~S}_{0}\right)$ and ${ }^{5}\left(\mathrm{~T}_{1} \mathrm{~T}_{1}\right)$, the $\omega \mathrm{B} 97 \mathrm{X}-\mathrm{D} / 6-31 \mathrm{G}(\mathrm{d})$ and $\mathrm{U} \omega \mathrm{B} 97 \mathrm{X}-\mathrm{D} / 6-31 \mathrm{G}(\mathrm{d})$ methods were used, respectively; for ${ }^{1}\left(S_{0} S_{1}\right)$, the TD- $\omega$ B97X-D/6$31 \mathrm{G}(\mathrm{d})$ method was used. ${ }^{28}$ Second, for the respective optimized geometries, the multiexcitonic states, including ${ }^{1}\left(\mathrm{~S}_{0} \mathrm{~S}_{0}\right),{ }^{1}\left(\mathrm{~S}_{0} \mathrm{~S}_{1}\right),{ }^{1}\left(\mathrm{~S}_{0} \mathrm{~S}_{1}\right){ }^{\prime}$, and ${ }^{1}\left(\mathrm{~T}_{1} \mathrm{~T}_{1}\right)$, were calculated based on a configuration interaction approach called the RAS-2SF method for four electrons in four orbitals ${ }^{44}$ [denoted as RAS(4,4)-2SF/6-31G(d)]. A detailed description of the geometry optimization and RAS-2SF calculation are given in Supporting Information. The geometry optimization and frequency analyses were performed with the Gaussian 16 program package $^{45}$ and the RAS-2SF calculations were performed with a code written by Casanova implemented in the Q-Chem program package. ${ }^{46}$ The methods of the quantum chemical calculations are described in detail in the Supporting Information. In the previous work, SF properties were calculated only for a ${ }^{1}\left(\mathrm{~S}_{0} \mathrm{~S}_{0}\right)$ geometry. ${ }^{39}$ Hence, if a molecule is largely deformed during $\mathrm{SF}$, the previous method would give 
unreliable SF rates. To consider effects of geometry deformation on SF properties, in this work, SF rates are calculated for ${ }^{1}\left(S_{0} S_{0}\right),{ }^{1}\left(S_{0} S_{1}\right)$, and ${ }^{5}\left(T_{1} T_{1}\right)$ geometries.

The nonradiative and radiative transition rates were calculated with the RAS(4,4)-2SF/6-31G(d) wave functions and the Fermi golden rule. The method of calculating the nonradiative transition rates has been described in detail elsewhere, ${ }^{39}$ and their validity has been demonstrated for intramolecular and intermolecular SF rates. ${ }^{39,42}$ The IC rate $\left(r_{\mathrm{IC}}\right)$ for an interstate transition can be written as the sum of contributions from all the individual vibrational modes: $r_{\mathrm{IC}}=$ $\sum_{m=1}^{M} r_{\mathrm{IC}, m}$, where $M$ is the total number of vibrational modes. $r_{\mathrm{IC}, m}$ can be expressed as

$$
\begin{aligned}
r_{\mathrm{IC}, m}= & \frac{2 \pi \hbar^{3}}{\left(E_{\mathrm{e}}^{\mathrm{f}}-E_{\mathrm{e}}^{\mathrm{i}}\right)^{2}} V_{m}^{2} P_{m} \\
V_{m}= & \left\langle\Phi^{\mathrm{f}}\left|\frac{\partial \hat{U}_{\mathrm{en}}}{\partial Q_{m}}\right| \Phi^{\mathrm{i}}\right\rangle \\
P_{m}= & \sinh \left(\frac{1}{2} \beta \hbar \omega_{m}\right) \sum_{v_{m}^{i}=0}^{\infty} \exp \left\{-\left(\frac{1}{2}+v_{m}\right) \beta \hbar \omega_{m}\right\} \\
& \times\left\{v_{m} D\left(\Delta E+\hbar \omega_{m}\right)+\left(v_{m}+1\right) D\left(\Delta E-\hbar \omega_{m}\right)\right\}
\end{aligned}
$$

where the suffixes $i$ and $f$ denote the initial and final states, respectively; the suffix $m$ denotes the $m$ th vibrational mode; $V_{m}$ denotes the vibronic coupling between the initial and final states owing to the $m$ th vibrational mode; $\Phi^{\mathrm{i}}$ and $\Phi^{\mathrm{f}}$ denote electronic wave functions of the initial and final states calculated using the RAS $(4,4)-2 S F / 6-31 G(d)$ method, respectively; $\hat{U}_{\text {en }}$ denotes the electron-nucleus potential, and $Q_{m}$ denotes the normal coordinate of the $m$ th vibrational mode; $E_{\mathrm{e}}^{\mathrm{f}}$ $-E_{\mathrm{e}}^{\mathrm{i}}$ denotes the Franck-Condon energy difference between the electronic energies of the initial and final states; $\Delta E$ also denotes the energy difference between the initial and final states but calculated from corrected RAS $(4,4)-2$ SF/6-31G(d) energies $^{39,42}$ (the method of the calculating corrected energies is described in the Supporting Information); $\beta$ denotes the inverse temperature $\left(\beta^{-1}=k_{\mathrm{B}} T\right.$, where $k_{\mathrm{B}}$ is the Boltzmann constant and $T$ is the temperature); and $\omega_{m}$ and $v_{m}$ denote the angular frequency and vibrational quantum number of the $m$ th vibrational mode, respectively; $D(x)=\frac{1}{\pi} \frac{\gamma}{x^{2}+\gamma^{2}}$ denotes the Lorentzian distribution function $(\gamma=\mathrm{fwhm} / 2$ and $\mathrm{fwhm}=600$ $\mathrm{cm}^{-1}$ ) describing dephasing effects owing to interactions with surrounding molecules in the solid state. ${ }^{42}$ The parameter $P_{m}$ contains information regarding the density of the final vibrational states. Equation 3 assumes the harmonic approximation for vibrational modes and same mode approximation. Under the assumptions, the radiative transition rate $\left(r_{\mathrm{em}}\right.$ : em denotes emission) can be expressed as $r_{\mathrm{em}}=\frac{4 \omega_{\mathrm{if}}{ }^{3}}{3 \hbar c^{3}} \mu_{\mathrm{if}}{ }^{2},{ }^{47}$ where $\omega_{\text {if }}=\frac{E_{\mathrm{e}}^{\mathrm{f}}-E_{\mathrm{e}}^{\mathrm{i}}}{\hbar}$ and $\mu_{\mathrm{if}}$ are the transition dipole moment between the initial and final states. $\omega_{\text {if }}$ and $\mu_{\text {if }}$ are calculated by the RAS(4,4)-2SF/6-31G(d) method.

\section{ASSOCIATED CONTENT}

\section{SI Supporting Information}

The Supporting Information is available free of charge at https://pubs.acs.org/doi/10.1021/acsomega.0c04809.
Standard nuclear orientations of the stable ${ }^{1}\left(\mathrm{~S}_{0} \mathrm{~S}_{0}\right)$, ${ }^{1}\left(\mathrm{~S}_{0} \mathrm{~S}_{1}\right)$, and ${ }^{5}\left(\mathrm{~T}_{1} \mathrm{~T}_{1}\right)$ geometries of 55BT and stable $\mathrm{S}_{0}$, $S_{1}$, and $T_{1}$ geometries of $\mathrm{Tc}$; tube representations of the stable geometries; Slater dominants mainly contributing to ${ }^{1}\left(\mathrm{~S}_{0} \mathrm{~S}_{0}\right),{ }^{1}\left(\mathrm{~S}_{0} \mathrm{~S}_{1}\right),{ }^{1}\left(\mathrm{~S}_{0} \mathrm{~S}_{1}\right)^{\prime}$, and ${ }^{1}\left(\mathrm{~T}_{1} \mathrm{~T}_{1}\right)$ of $55 \mathrm{BT}$ calculated for its stable ${ }^{1}\left(S_{0} S_{0}\right),{ }^{1}\left(S_{0} S_{1}\right)$, and ${ }^{5}\left(T_{1} T_{1}\right)$ geometries; calculated excitation energies of ${ }^{1}\left(S_{0} S_{1}\right)$, ${ }^{1}\left(\mathrm{~S}_{0} \mathrm{~S}_{1}\right)^{\prime}$, and ${ }^{1}\left(\mathrm{~T}_{1} \mathrm{~T}_{1}\right)$ and corrected excitation energies; complete energy diagram of 55BT showing its stable ${ }^{1}\left(S_{0} S_{0}\right),{ }^{1}\left(S_{0} S_{1}\right)$, and ${ }^{5}\left(T_{1} T_{1}\right)$ geometries; interstate vibronic-coupling constants between ${ }^{1}\left(\mathrm{~T}_{1} \mathrm{~T}_{1}\right)$ and ${ }^{1}\left(S_{0} S_{1}\right)$ with respect to normal coordinates of 55BT calculated at the stable ${ }^{1}\left(S_{0} S_{1}\right)$ geometry; And calculation method of the $\mathrm{T}_{1}$ energies of 55BT and $\mathrm{Tc}$ (PDF)

\section{AUTHOR INFORMATION}

\section{Corresponding Author}

Hironori Kaji - Institute for Chemical Research, Kyoto

University, Uji, Kyoto 611-0011, Japan; 으이.org/0000-

0002-5111-3852; Email: kaji@scl.kyoto-u.ac.jp

\section{Authors}

Katsuyuki Shizu - Institute for Chemical Research, Kyoto University, Uji, Kyoto 611-0011, Japan; —orcid.org/00000002-1835-0418

Chihaya Adachi - Center for Organic Photonics and Electronics Research (OPERA) and International Institute for Carbon Neutral Energy Research (WPI-I2CNER), Kyushu University, Fukuoka 819-0395, Japan; Japan Science and Technology Agency (JST), ERATO, Adachi Molecular Exciton Engineering Project, Fukuoka 819-0395, Japan; (1) orcid.org/0000-0001-6117-9604

Complete contact information is available at:

https://pubs.acs.org/10.1021/acsomega.0c04809

\section{Author Contributions}

K.S. carried out all the theoretical calculations. H.K. and C.A. planned and supervised the project. All authors contributed to the writing of this manuscript. All authors have given approval to the final version of the manuscript.

Notes

The authors declare no competing financial interest.

\section{ACKNOWLEDGMENTS}

The quantum chemical calculations using the Gaussian 16 and Q-Chem program packages were performed using the SuperComputer System, Institute for Chemical Research, Kyoto University. This work was supported by the Japan Science and Technology Agency (JST), ERATO, Adachi Molecular Exciton Engineering Project, under JST ERATO grant number JPMJER1305, Japan. This work was also supported by JSPS KAKENHI grant numbers JP20H05840 (Grant-in-Aid for Transformative Research Areas, "Dynamic Exciton"), 17H01231, 17K14529, and 19K05629 and JSPS Core-to-Core Program. We thank Andrew Jackson, PhD, from the Edanz Group (https://en-author-services.edanzgroup. com/ac) for editing a draft of this manuscript.

\section{ABBREVIATIONS}

fwhm, full-width at half-maximum; IC, internal conversion; NIR, near-infrared; OLED, organic light-emitting diode; RAS, 
restricted active space; RAS-2SF, RAS double spin-flip; SF, singlet fission

\section{REFERENCES}

(1) Smith, M. B.; Michl, J. Singlet Fission. Chem. Rev. 2010, 110, 6891-6936.

(2) Smith, M. B.; Michl, J. Recent Advances in Singlet Fission. Annu. Rev. Phys. Chem. 2013, 64, 361-386.

(3) Lee, J.; Jadhav, P.; Baldo, M. A. High Efficiency Organic Multilayer Photodetectors Based on Singlet Exciton Fission. Appl. Phys. Lett. 2009, 95, 033301.

(4) Congreve, D. N.; Lee, J.; Thompson, N. J.; Hontz, E.; Yost, S. R.; Reusswig, P. D.; Bahlke, M. E.; Reineke, S.; Van Voorhis, T.; Baldo, M. A. External Quantum Efficiency above $100 \%$ in a Singlet-ExcitonFission-Based Organic Photovoltaic Cell. Science 2013, 340, 334.

(5) Nagata, R.; Nakanotani, H.; Potscavage, W. J.; Adachi, C. Exploiting Singlet Fission in Organic Light-Emitting Diodes. Adv. Mater. 2018, 30, 1801484.

(6) Qian, G.; Wang, Z. Y. Near-Infrared Organic Compounds and Emerging Applications. Chem.-Asian J. 2010, 5, 1006-1029.

(7) Pansare, V. J.; Hejazi, S.; Faenza, W. J.; Prud'homme, R. K. Review of Long-Wavelength Optical and Nir Imaging Materials: Contrast Agents, Fluorophores, and Multifunctional Nano Carriers. Chem. Mater. 2012, 24, 812-827.

(8) Zampetti, A.; Minotto, A.; Cacialli, F. Near-Infrared (Nir) Organic Light-Emitting Diodes (Oleds): Challenges and Opportunities. Adv. Funct. Mater. 2019, 29, 1807623.

(9) Swenberg, C. E.; Stacy, W. T. Bimolecular Radiationless Transitions in Crystalline Tetracene. Chem. Phys. Lett. 1968, 2, 327-328.

(10) Tomkiewicz, Y.; Groff, R. P.; Avakian, P. Spectroscopic Approach to Energetics of Exciton Fission and Fusion in Tetracene Crystals. J. Chem. Phys. 1971, 54, 4504-4507.

(11) Burdett, J. J.; Müller, A. M.; Gosztola, D.; Bardeen, C. J. Excited State Dynamics in Solid and Monomeric Tetracene: The Roles of Superradiance and Exciton Fission. J. Chem. Phys. 2010, 133, 144506.

(12) Burdett, J. J.; Gosztola, D.; Bardeen, C. J. The Dependence of Singlet Exciton Relaxation on Excitation Density and Temperature in Polycrystalline Tetracene Thin Films: Kinetic Evidence for a Dark Intermediate State and Implications for Singlet Fission. J. Chem. Phys. 2011, 135, 214508.

(13) Burdett, J. J.; Bardeen, C. J. The Dynamics of Singlet Fission in Crystalline Tetracene and Covalent Analogs. Acc. Chem. Res. 2013, 46, $1312-1320$.

(14) Piland, G. B.; Burdett, J. J.; Dillon, R. J.; Bardeen, C. J. Singlet Fission: From Coherences to Kinetics. J. Phys. Chem. Lett. 2014, 5, 2312-2319.

(15) Piland, G. B.; Burdett, J. J.; Hung, T.-Y.; Chen, P.-H.; Lin, C.F.; Chiu, T.-L.; Lee, J.-H.; Bardeen, C. J. Dynamics of Molecular Excitons near a Semiconductor Surface Studied by Fluorescence Quenching of Polycrystalline Tetracene on Silicon. Chem. Phys. Lett. 2014, 601, 33-38.

(16) Roberts, S. T.; McAnally, R. E.; Mastron, J. N.; Webber, D. H.; Whited, M. T.; Brutchey, R. L.; Thompson, M. E.; Bradforth, S. E. Efficient Singlet Fission Discovered in a Disordered Acene Film. J. Am. Chem. Soc. 2012, 134, 6388-6400.

(17) Mastron, J. N.; Roberts, S. T.; McAnally, R. E.; Thompson, M. E.; Bradforth, S. E. Aqueous Colloidal Acene Nanoparticles: A New Platform for Studying Singlet Fission. J. Phys. Chem. B 2013, 117, 15519-15526.

(18) Ryasnyanskiy, A.; Biaggio, I. Triplet Exciton Dynamics in Rubrene Single Crystals. Phys. Rev. B: Condens. Matter Mater. Phys. 2011, 84, 193203.

(19) Ma, L.; Zhang, K.; Kloc, C.; Sun, H.; Michel-Beyerle, M. E.; Gurzadyan, G. G. Singlet Fission in Rubrene Single Crystal: Direct Observation by Femtosecond Pump-Probe Spectroscopy. Phys. Chem. Chem. Phys. 2012, 14, 8307-8312.

(20) Ma, L.; Zhang, K.; Kloc, C.; Sun, H.; Soci, C.; Michel-Beyerle, M. E.; Gurzadyan, G. G. Fluorescence from Rubrene Single Crystals:
Interplay of Singlet Fission and Energy Trapping. Phys. Rev. B: Condens. Matter Mater. Phys. 2013, 87, No. 201203(R).

(21) Jankus, V.; Snedden, E. W.; Bright, D. W.; Arac, E.; Dai, D.; Monkman, A. P. Competition between Polaron Pair Formation and Singlet Fission Observed in Amorphous Rubrene Films. Phys. Rev. B: Condens. Matter Mater. Phys. 2013, 87, 224202.

(22) Piland, G. B.; Burdett, J. J.; Kurunthu, D.; Bardeen, C. J. Magnetic Field Effects on Singlet Fission and Fluorescence Decay Dynamics in Amorphous Rubrene. J. Phys. Chem. C 2013, 117, 12241236.

(23) Gieseking, B.; Schmeiler, T.; Müller, B.; Deibel, C.; Engels, B.; Dyakonov, V.; Pflaum, J. Effects of Characteristic Length Scales on the Exciton Dynamics in Rubrene Single Crystals. Phys. Rev. B: Condens. Matter Mater. Phys. 2014, 90, 205305.

(24) Ward, K. A.; Richman, B. R.; Biaggio, I. Nanosecond Pump and Probe Observation of Bimolecular Exciton Effects in Rubrene Single Crystals. Appl. Phys. Lett. 2015, 106, 223302.

(25) Miyata, K.; Kurashige, Y.; Watanabe, K.; Sugimoto, T.; Takahashi, S.; Tanaka, S.; Takeya, J.; Yanai, T.; Matsumoto, Y. Coherent Singlet Fission Activated by Symmetry Breaking. Nat. Chem. 2017, 9, 983.

(26) Müller, A. M.; Avlasevich, Y. S.; Müllen, K.; Bardeen, C. J. Evidence for Exciton Fission and Fusion in a Covalently Linked Tetracene Dimer. Chem. Phys. Lett. 2006, 421, 518-522.

(27) Müller, A. M.; Avlasevich, Y. S.; Schoeller, W. W.; Müllen, K.; Bardeen, C. J. Exciton Fission and Fusion in Bis(Tetracene) Molecules with Different Covalent Linker Structures. J. Am. Chem. Soc. 2007, 129, 14240-14250.

(28) Vallett, P. J.; Snyder, J. L.; Damrauer, N. H. Tunable Electronic Coupling and Driving Force in Structurally Well-Defined Tetracene Dimers for Molecular Singlet Fission: A Computational Exploration Using Density Functional Theory. J. Phys. Chem. A 2013, 117, 10824-10838.

(29) Alguire, E. C.; Subotnik, J. E.; Damrauer, N. H. Exploring NonCondon Effects in a Covalent Tetracene Dimer: How Important Are Vibrations in Determining the Electronic Coupling for Singlet Fission? J. Phys. Chem. A 2015, 119, 299-311.

(30) Damrauer, N. H.; Snyder, J. L. Symmetry-Directed Control of Electronic Coupling for Singlet Fission in Covalent Bis-Acene Dimers. J. Phys. Chem. Lett. 2015, 6, 4456-4462.

(31) Cook, J. D.; Carey, T. J.; Damrauer, N. H. Solution-Phase Singlet Fission in a Structurally Well-Defined Norbornyl-Bridged Tetracene Dimer. J. Phys. Chem. A 2016, 120, 4473-4481.

(32) Carey, T. J.; Snyder, J. L.; Miller, E. G.; Sammakia, T.; Damrauer, N. H. Synthesis of Geometrically Well-Defined Covalent Acene Dimers for Mechanistic Exploration of Singlet Fission. J. Org. Chem. 2017, 82, 4866-4874.

(33) Feng, X.; Krylov, A. I. On Couplings and Excimers: Lessons from Studies of Singlet Fission in Covalently Linked Tetracene Dimers. Phys. Chem. Chem. Phys. 2016, 18, 7751-7761.

(34) Korovina, N. V.; Das, S.; Nett, Z.; Feng, X.; Joy, J.; Haiges, R.; Krylov, A. I.; Bradforth, S. E.; Thompson, M. E. Singlet Fission in a Covalently Linked Cofacial Alkynyltetracene Dimer. J. Am. Chem. Soc. 2016, 138, 617-627.

(35) Korovina, N. V.; Joy, J.; Feng, X.; Feltenberger, C.; Krylov, A. I.; Bradforth, S. E.; Thompson, M. E. Linker-Dependent Singlet Fission in Tetracene Dimers. J. Am. Chem. Soc. 2018, 140, 1017910190.

(36) Matsui, Y.; Kawaoka, S.; Nagashima, H.; Nakagawa, T.; Okamura, N.; Ogaki, T.; Ohta, E.; Akimoto, S.; Sato-Tomita, A.; Yagi, S.; et al. Exergonic Intramolecular Singlet Fission of an AdamantaneLinked Tetracene Dyad Via Twin Quintet Multiexcitons. J. Phys. Chem. C 2019, 123, 18813-18823.

(37) Liu, H.; Wang, R.; Shen, L.; Xu, Y.; Xiao, M.; Zhang, C.; Li, X. A Covalently Linked Tetracene Trimer: Synthesis and Singlet Exciton Fission Property. Org. Lett. 2017, 19, 580-583.

(38) Kim, J.; Teo, H. T.; Hong, Y.; Oh, J.; Kim, H.; Chi, C.; Kim, D. Multiexcitonic Triplet Pair Generation in Oligoacene Dendrimers as 
Amorphous Solid-State Miniatures. Angew. Chem., Int. Ed. 2020, 59, 21247.

(39) Shizu, K.; Adachi, C.; Kaji, H. Effect of Vibronic Coupling on Correlated Triplet Pair Formation in the Singlet Fission Process of Linked Tetracene Dimers. J. Phys. Chem. A 2020, 124, 3641-3651.

(40) Toyota, S.; Miyaji, R.; Yamamoto, Y.; Inoue, M.; Wakamatsu, K.; Iwanaga, T. An Enantiopure 5,5'-Bitetracene. Eur. J. Org. Chem. 2015, 2015, 7648-7651.

(41) Turro, N. J.; Ramamurthy, V.; Scaiano, J. C. Modern Molecular Photochemistry of Organic Molecules; University Science Books: Sausalito, 2010.

(42) Shizu, K.; Adachi, C.; Kaji, H. Visual Understanding of Vibronic Coupling and Quantitative Rate Expression for Singlet Fission in Molecular Aggregates. Bull. Chem. Soc. Jpn. 2020, 93, $1305-1313$.

(43) Mackie, C. J.; Candian, A.; Huang, X.; Maltseva, E.; Petrignani, A.; Oomens, J.; Buma, W. J.; Lee, T. J.; Tielens, A. G. G. M. The Anharmonic Quartic Force Field Infrared Spectra of Three Polycyclic Aromatic Hydrocarbons: Naphthalene, Anthracene, and Tetracene. J. Chem. Phys. 2015, 143, 224314.

(44) Casanova, D.; Slipchenko, L. V.; Krylov, A. I.; Head-Gordon, M. Double Spin-Flip Approach within Equation-of-Motion Coupled Cluster and Configuration Interaction Formalisms: Theory, Implementation, and Examples. J. Chem. Phys. 2009, 130, 044103.

(45) Frisch, M. J.; Trucks, G. W.; Schlegel, H. B.; Scuseria, G. E.; Robb, M. A.; Cheeseman, J. R.; Scalmani, G.; Barone, V.; Petersson, G. A.; Nakatsuji, H.; et al. Gaussian 16, Rev. B.01, Wallingford, CT, 2016.

(46) Shao, Y.; Gan, Z.; Epifanovsky, E.; Gilbert, A. T. B.; Wormit, M.; Kussmann, J.; Lange, A. W.; Behn, A.; Deng, J.; Feng, X.; et al. Advances in Molecular Quantum Chemistry Contained in the QChem 4 Program Package. Mol. Phys. 2015, 113, 184-215.

(47) Toptygin, D. Effects of the Solvent Refractive Index and Its Dispersion on the Radiative Decay Rate and Extinction Coefficient of a Fluorescent Solute. J. Fluoresc. 2003, 13, 201-219. 